\title{
GLOSSARY OF COMMONLY USED TERMS AND ABBREVIATIONS
}

ACEs

ACL

ADA

ADL

ADRCs

AIMS

ARD

ASAP

BBW

BI

BIMS

BP

$\mathrm{C}^{-}$

CAA adverse childhood experiences

Administration for Community Living

Americans With Disabilities Act

activities of daily living

Aging and Disability Resource Centers

Abnormal Involuntary Movement Inventory Scale Assessment reference date-A2300

- Use entire $24 \mathrm{hrs}$ of the ARD to assess resident

- Begins at 12:00 a.m. and ends at 11:59 p.m. A2300: Assessment Reference Date

- Midnight to Midnight

- $\quad$ ARD of October 01,2010 or later $=$ MDS 3.0

- $\mathrm{ARD}$ of September 30, 2010 or before = MDS 2.0

assessment submission and processing

black-box warning

brain injury

Brief Interview for Mental Status

blood pressure

with

Care Area Assessment-Trigger on the Minimum Data Set 3.0 replaces the Minimum Data Set 2.0 RAP process and includes:

- care area triggers (CATs) similar to RAP triggers

- review of care areas uses evidence-based tools, expert-endorsed research, clinical practice guidelines, and other resources 

CAM Confusion Assessment Method
care plans all residents have an individual interdisciplinary plan of care for all their problems and needs. This includes identification of a specific problem or need; a succinct, measurable, time-specific goal; and intervention(s) by team members.

case mix

reimbursement services that reflects the specific patient care needs of the residents

case mix system database storing all Management Minutes Questionnaires data that will be used for administrative and quality monitoring purposes

CASPER Certification and Survey Provider Enhanced Reports (Minimum Data Set reporting system)

CMS

CNA

c/o

COPD

CSA

$\mathrm{d} / \mathrm{c}$

$\mathrm{D} / \mathrm{C}$ Centers for Medicare \& Medicaid Services certified nursing assistant complaints of chronic obstructive pulmonary disease childhood sexual abuse discharge discontinue

DDS

Developmental Disability Services

DHS

Department of Human Services

DMAT

Disaster Medical Assistance Team

DNR

do not resuscitate

DOD

Department of Defense

DOT

Department of Transportation

DPH

Department of Public Health

DPOA

durable power of attorney

DRA

Deficit Reduction Act

DSM

DTI

Diagnostic and Statistical Manual of Mental Disorders deep tissue injury

ECHO

Elder Cottage Housing Opportunity

EPSDT

Early and Periodic Screening, Diagnosis, and Treatment

fax facsimile

FBS fasting blood sugar

FDA U.S. Food and Drug Administration

FEMA fx Federal Emergency Management Agency fracture 
GOSS

HCBS

HCFA

HD

HHAs

HIPAA

HMO

hs

Hx

I \& $\mathrm{O}$

ICF

IDT

IFMC

IM

inc

IPT

JCAHO

$\lg$

LOC

LSW

LTC

LTCOP

Managed

Minutes

MDS

Medicaid goal-oriented social service

home- and community-based services

Health Care Financing Administration

Huntington's disease

home health agencies

Health Insurance Portability and Accountability

Act of 1996

health maintenance organization

hour of sleep or bedtime

history

intake and output

intermediate care facilities

interdisciplinary team

Iowa Foundation for Medical Care

intramuscular

incontinent

Iowa Pain Thermometer

Joint Commission on Accreditation of Healthcare

Organizations

large

level of care

licensed social worker

long-term care

Long-Term Care Ombudsman Program

a method of measuring case mix by assigning weights to discrete caregiving activities or to characteristics of patients found to require given amounts of care. The Managed Minutes Data System is the organized procedure for collecting, storing, and analyzing this information.

Minimum Data Set (3.0). This form provides a core set of clinical and functional status screening elements that form the foundation of the comprehensive assessment. Title XIX of the Social Security Act. This federal and state-funded grant program and state-administrated healthcare program provides indigent people (both young and old) with access to healthcare. Each state sets individual income and asset guidelines in order for individuals to qualify for the program. 


\section{Medicare}

Parts A and B Title XVIII of the Social Security Act. Parts A \& BPremiums are paid for by participants as this is both an entitlement and an insurance program. Individuals qualify by having worked within the Social Security system or by being disabled and meeting qualifications.

MDS

MFP Minimum Data Set (2.0). This form provides a core set of clinical and functional status screening elements that form the foundation of the comprehensive assessment

MGL Money Follows the Person

Massachusetts General Law

MI

MR

NASW

NCDs

NCEA

NDMS

nf

NGT

NH

noc

NPO

NRP

NRS

OASIS

mental illness

mental retardation

National Association of Social Workers

neurocognitive disorders

National Center on Elder Abuse

National Disaster Medical System

nursing facility

nasal gastric tube

nursing home

night

nothing by mouth

National Response Plan

Numeric Rating Scale

Outcome and Assessment Information Set

OBRA

Omnibus Reconciliation Act of 1987. Implemented across the country in 1989, making enormous, sweeping changes and additions to the regulation of long-term care.

OIG Office of Inspector General

OMRA Other Medicare Required Assessment. Medicare PPS assessment for start or end of therapy and resident still requires skilled nursing

OSCAR

Standard Reports \#3 and \#4 from HCFA's Online Survey, Certification and Reporting System. Report \#3 gives the state surveyor a history of the facility in profile per the last four previous surveys as well as additional complaints, problems, and corrections. Report \#4 provides the surveyors with the facility's physical plant problems and corrections, if any. 
OT

PASRR

PDPM

PHQ-9

PIP

PNA

po

POA

PPE

PPS

prn

pt

PT

PU

QAA

QAPI

qd

qh

qid

QIO

QM

QMB/QIP

qod

QIES

RAI

occupational therapy

The OBRA acronym for Preadmission Screening and

Resident Review. The requirements of OBRA $\$ 483.100-$ $\$ 483.136$ govern the state’s responsibility for preadmission screening and annual resident review of individuals with mental illness and mental retardation.

Patient Driven Payment Model

Patient Health Questionnaire, Nine Questions

performance improvement projects

personal needs account. This is money left in an account in the facility for the resident. Medicaid currently provides eligible residents in nursing facilities with a monthly amount determined by each individual state. by mouth power of attorney personal protective equipment

Prospective Payment System. Payment system for facilities participating in Medicare. This means that, the facilities based on preset rates and codes, the facilities are paid by Medicare in a lump sum for care of eligible residents.

as needed

patient

physical therapy

pressure ulcer

quality assessment and assurance

Quality Assurance and Performance Improvement

every day

every hour

four times a day

quality-improvement organization

quality measures

Qualifying Medicare Beneficiary/Qualifying Individual Program

every other day

Quality Improvement and Evaluation System

Resident Assessment Instrument. A standard, structured approach used to apply a problem identification model in long-term care facilities

Resident Assessment Protocol 
RCCs

RN

RUGS-s III

$\mathbf{R x}$

$s$

SAMHSA

SB

SCSA

SNF

SOAP

SOB

SS

SSAs

SSI

ST

TID

TJC

tx

UTI

vd

VNA

$\mathrm{w} / \mathrm{c}$ residential care communities

registered nurse

Resource Utilization Groups. This is a patient classification system that identifies the relative costs (resource costs) of providing care for different types of patients based upon their resource use.

medication

without

Substance Abuse and Mental Health Services Administration

swing bed unit

significant change in status assessment

skilled nursing facility

subjective, objective, assessment, plan

shortness of breath

Social Security

state survey agencies

Supplemental Security Income

speech therapy

three times a day

The Joint Commission

treatment

urinary tract infection

voided

Visiting Nurse Association

wheelchair 